Supporting information

\title{
High-throughput single-cell extracellular vesicle secretion analysis on the desktop scanner without cell counting
}

Fengjiao Zhu ${ }^{1,2}$, Yahui Ji ${ }^{1}$, Linmei $\mathrm{Li}^{1}$, Xue Bai ${ }^{1}$, Xianming Liu ${ }^{1}$, Yong Luo ${ }^{3}$, Tingjiao Liu ${ }^{4}$, Bingcheng $\mathrm{Lin}^{1}$, Yao Lu*1

${ }^{1}$ Department of Biotechnology, Dalian Institute of Chemical Physics, Chinese Academy of Sciences, Dalian, 116023, China ${ }^{2}$ University of Chinese Academy of Sciences, Beijing, 100049, China

${ }^{3}$ State Key Laboratory of Fine Chemicals, Department of Chemical Engineering, Dalian University of Technology, Dalian, 116024, China

${ }^{4}$ College of Stomatology, Dalian Medical University, Dalian, 116044, China

*Correspondence should be addressed to Y. Lu (luyao@dicp.ac.cn) 
This supporting information includes: Experimental methods; The design of the microwell array chip; Characterization of antibody, exosome-depleted FBS, sliver staining, desktop scanner and EV concentration in culture medium; Cell density optimization; The calibration curves for ${ }^{\mathrm{CD} 9+\mathrm{CD} 63+} \mathrm{EVs}$ and ${ }^{\mathrm{CD} 63+} \mathrm{EV}$; The maximum EV capturing capacity of the CD63 antibody glass slide; Information for antibodies and patients.

Table of contents

Figure S1. The design of the high throughput microwell array chip. S-4

Figure S2. Characterization of protein immobilization on a poly-L-lysine glass slide. S-5

Figure S3. Verification of antibody specificity. S-6

Figure S4. Validation of detection specificity with TF-1 cells. S-7

Figure S5. A comparison of exosome-depleted FBS at different ultracentrifuge times. S-8

Figure S6. SEM characterization of antibody captured EVs (fluorescence detection region). S-9

Figure S7. The time-course monitoring of silver staining showed that the largest $\mathrm{S} / \mathrm{N}$ was obtained after 8 min of silver enhancement. S-10

Figure S8. Characterization of desktop scanner for detection. S-11

Figure S9. Cell viability characterization. S-12

Figure S10. Cell density optimization. S-13

Figure S11. Calculation of the theoretical value of the number of captured cells. S-14

Figure S12. Characterization of EV concentration in culture medium with NTA (Particle Metrix ZetaView ${ }^{\circledR}$, Germany. Measurement Mode: Size Distribution 3 Cycles 11 Positions). S-15

Figure S13. The calibration curves for ${ }^{\mathrm{CD} 9}+\mathrm{CD} 63+\mathrm{EVs}$ and ${ }^{\mathrm{CD} 63+} \mathrm{EVs}$ using EV standard (Biovision, US) by immunofluorescence. S-16

Figure S14: Characterization of the maximum EV capturing capacity of the CD63 antibody glass slide. S-17

Table S1. Summary of reagents used. S-18

Table S2. The patients' medical records. S-18 
Preparation of antibody-coated glass slide. The capture antibody (100 $\mu \mathrm{L}, 20 \mu \mathrm{g} / \mathrm{mL})$ (Table $\mathrm{S} 1)$ was immobilized on a poly-L-lysine coated glass slide (Thermo, America) by incubating at $4{ }^{\circ} \mathrm{C}$ in a wet box overnight. After the antibody coating, the glass slide was blocked with $3 \%(\mathrm{w} / \mathrm{v})$ BSA (Gentihold, China) for $1 \mathrm{~h}$ to reduce non-specific adsorption. Afterward, it was washed with phosphate-buffered saline (DPBS) (Coolaber, China), 50\% (v/v) DPBS, and distilled water ( DI water), sequentially. The antibody-coated glass slide was spun dry in a slide centrifuge and stored at $4{ }^{\circ} \mathrm{C}$ before use.

OSCC Patient Samples. Human OSCC patient samples were obtained from the Affiliated Hospital of Dalian Medical University. This study was carried out under the approval of the Ethics Committee of Dalian Medical University. We used the enzyme digestion method to extract OSCC cells from tissues. Briefly, fresh patient tissue was first minced into $\sim 1 \mathrm{~mm}^{3}$ piece. After washing with DPBS containing $2 \%$ antibiotics over and over again, the tissue was then detached with $0.25 \%$ trypsin $-0.02 \%$ EDTA for $30 \mathrm{~min}$ at $37{ }^{\circ} \mathrm{C}$. The pellet was then detached with collagenase I on the shaker until it became flocculent, which would spread across the culture dish coated with collagenase I. The culture dish was placed in a $37{ }^{\circ} \mathrm{C}, 5 \% \mathrm{CO} 2$ incubator for $1 \mathrm{~h}$. DMEM/HG complete medium was added and changed periodically until the cells became confluent in the culture dish. Afterward, the cells isolated from living tissue were used for the experiment.

Cell culture. Human oral squamous carcinoma cells (SCC25) (American Type Culture Collection) were cultured in MEM (Hyclone, America) complete medium containing 10\% (v/v) fetal bovine serum (PAN, Germany), which was ultracentrifuged for $2 \mathrm{~h}$ at $100,000 \times \mathrm{g}$ (Type 90 Ti rotor, Beckman Coulter) at $4{ }^{\circ} \mathrm{C}$ to deplete exosomes, $100 \mathrm{U} / \mathrm{mL}$ of penicillin G sodium and $100 \mathrm{U} / \mathrm{mL}$ of streptomycin (Hyclone, America) and 1\% (v/v) MEM Non-Essential Amino Acid (Life Technologies, America). Primary human oral squamous carcinoma cells were cultured in DMEM/High glucose (Hyclone, America) complete medium with similar conditions as for SCC25. The cytokine-dependent erythroleukemia cell line TF-1 (American Type Culture Collection) was cultured in RPMI 1640 medium supplemented with 10\% (v/v) FBS (PAN, Germany), $100 \mathrm{U} / \mathrm{mL}$ of penicillin G sodium and $100 \mathrm{U} / \mathrm{mL}$ of streptomycin (Hyclone, America), and 2 $\mathrm{ng} / \mathrm{ml}$ granulocyte-macrophage colony-stimulating factor (GM-CSF) (R\&D, America). Cells were cultured in an incubator at $37{ }^{\circ} \mathrm{C}$ and $5 \% \mathrm{CO} 2$. Cells were subcultured while they reached confluence, which was trypsinized with $0.25 \%$ trypsin (Coolaber, China) $+0.02 \%$ EDTA (Coolaber, China) for 3 min before centrifuging at $1000 \mathrm{rpm}$ for $5 \mathrm{~min}$. After resuspending in a fresh medium, the cells are ready for experiments.

Single-cell EV secretion assay using fluorescence-based immunoassay. The procedure for trapping and incubating cells with microchambers has been described in detail above. The standard ELISA procedure was used for EV detection. Firstly, biotinylated anti-human CD63 was pipetted onto the glass slide and incubated for $1 \mathrm{~h}$ at room temperature. After the glass slide was thoroughly washed three times with $1 \%(\mathrm{w} / \mathrm{v})$ BSA in DPBS, Streptavidin-APC (1:100 dilution in 1\% BSA, Thermo, America) was pipetted onto the glass slide and incubated for $0.5 \mathrm{~h}$. After washing with DPBS and DI water, it was spun dry in a slide centrifuge and imaged with GenePix 4300A scanner. 


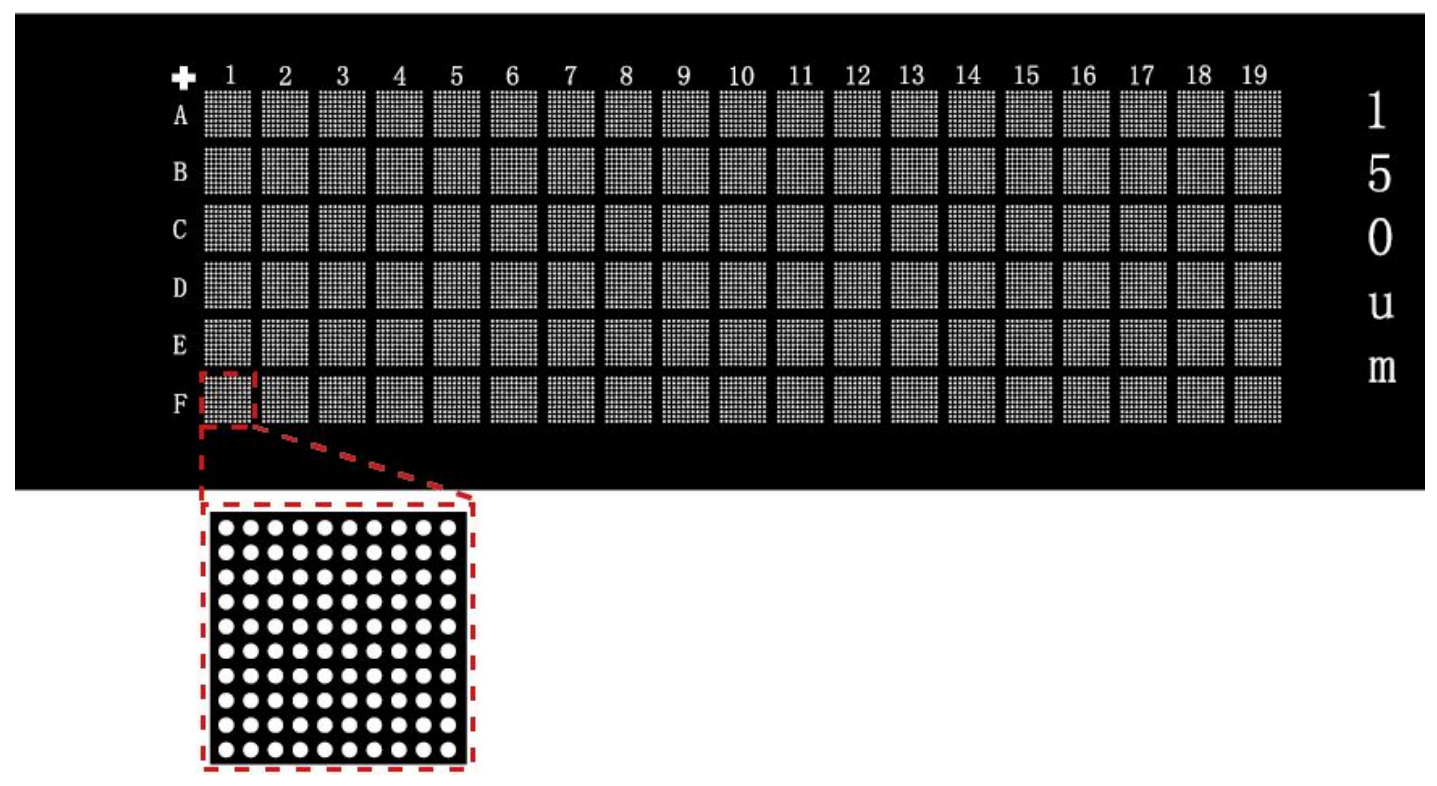

Figure S1. The design of the high throughput microwell array chip. PDMS microwell array chip was fabricated with soft lithography, which contains 11,400 microwells $(150 \mu \mathrm{m}$ in diameter and $70 \mu \mathrm{m}$ in depth, with a spacing of $100 \mu \mathrm{m}$ between neighboring microwells). 

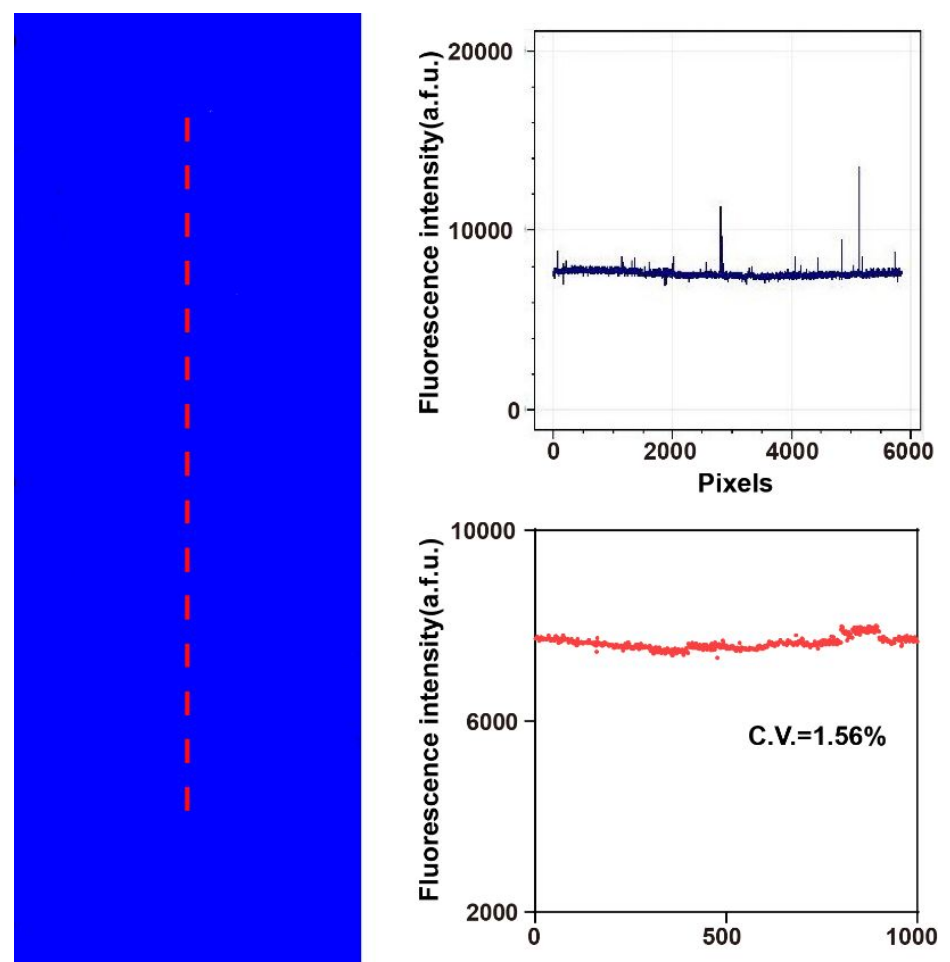

Figure S2. Characterization of protein immobilization on a poly-L-lysine glass slide. We used FITC-BSA as a model protein to characterize the immobilization uniformity across the glass side since both FITC-BSA and antibody would be immobilized onto a poly-L-lysine glass slide through electrostatic interaction. Therefore, the uniformity of protein immobilization on the poly-L-lysine glass slide can be assessed. Fluorescein isothiocyanate labeled BSA (100 $\mu \mathrm{L}, 0.25$ $\mathrm{mg} / \mathrm{mL}$ ) was immobilized on a poly-L-lysine glass slide. After blocking and washing, the slide was scanned by GenePix 4300A and analyzed by GenePix Pro 7. 1000 circular spots (150 $\mu \mathrm{m}$ diameter) were selected randomly across the glass slide to extract their fluorescence signal intensities, which revealed that FITC-BSA was uniformly coated on a poly-Llysine glass slide $(\mathrm{C} . \mathrm{V} .=1.56 \%)$. Therefore, it can be inferred that the poly-L-lysine glass slide can be used as an excellent substrate to immobilize capture antibodies uniformly. And the detection of EVs across the glass slide would not be affected by the uniformity of the capture antibodies. 


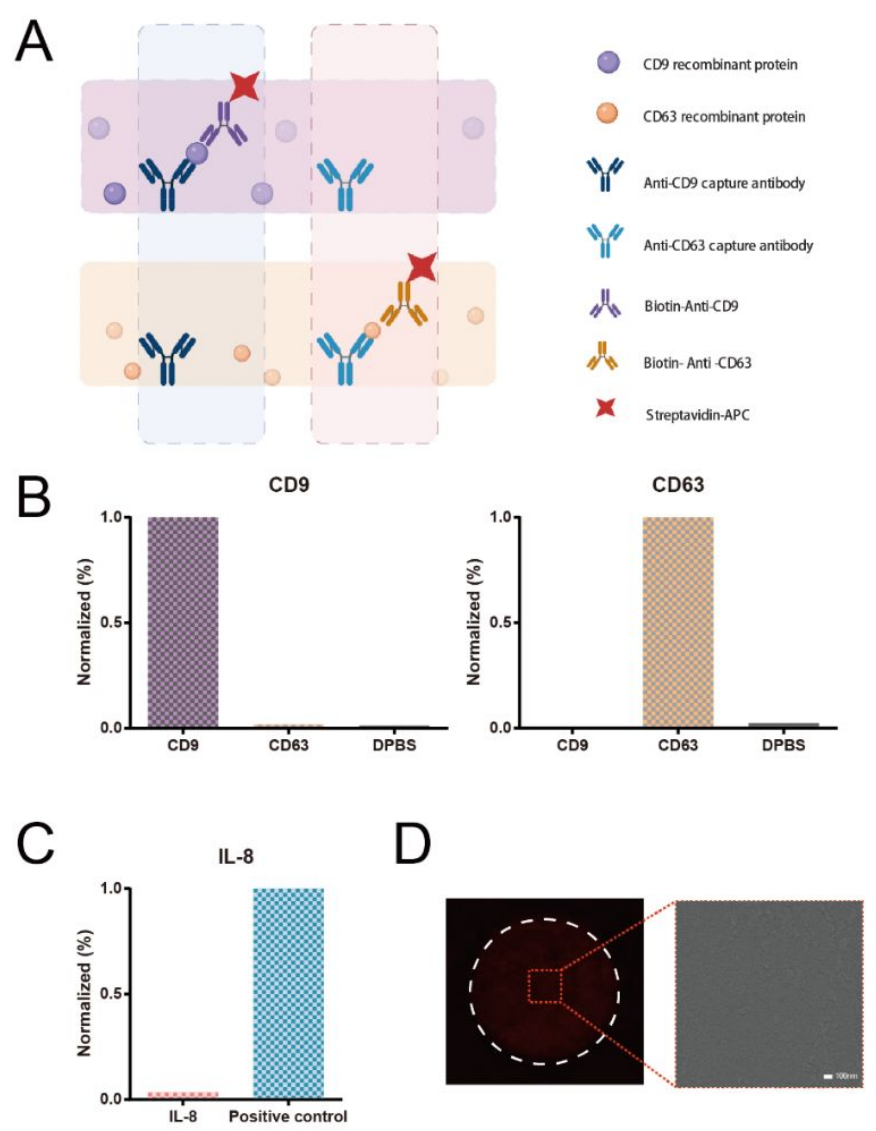

Figure S3. Verification of antibody specificity. (A) Schematic illustrating the cross-reactivity test, which is very similar to sandwich ELISA detection procedures. (B) Crosstalk test between CD9 and CD63 antibodies with recombinant proteins. The CD9 and CD63 capture antibodies $(2 \mu \mathrm{L}, 100 \mu \mathrm{g} / \mathrm{mL})$ were immobilized on a poly-L-lysine coated glass slide by incubating at $4{ }^{\circ} \mathrm{C}$ in a wet box overnight to form antibody stripes. Both CD9 and CD63 antibodies used in this study are monoclonal antibodies to reduce cross-reaction. Afterward, CD9 (Prospe, Israel) and CD63 (Raybiotch, America) recombinant proteins were added and incubated for 4 hours at $25^{\circ} \mathrm{C}$, respectively. After blocking and washing, the glass slide was incubated with a mixture of biotin-CD9 and biotin-CD63 (1:200 dilution, Biolegend) for $1 \mathrm{~h}$ and stained with streptavidin-APC (1:100 dilution, eBioscience) for $30 \mathrm{~min}$. The glass slide was washed with DPBS, DPBS/DI water(v/v=1/1), and DI water sequentially. Then it was spun dry in a slide centrifuge. The fluorescence results were read and analyzed with GenePix scanner and software. The signal intensity was normalized into a percentage. The fluorescence intensity after normalization reveals that the crosstalk between CD9 and CD63 antibody detection was less than 5\%. (C) Crosstalk assay revealed that a cytokine antibody would not capture EV. IL-8 capture antibody (IL-8 is not expressed on the surface of the EV) was used to capture EV to evaluate false-positive signals caused by non-specific adsorption. Meanwhile, CD81 capture antibody was used to capture EV as a positive control. SCC25 culture medium containing EVs and were added and incubated. After blocking and washing, the glass slide was incubated with biotinCD63 and stained with streptavidin-APC to form a fluorescence region as a control. The fluorescence signal intensity was read by GenePix 4300A and analyzed by GenePix Pro 7. (D) SEM image confirmed EVs and cell debris were not captured by IL-8 capture antibody on the antibody-coated spot (scale bar is $100 \mathrm{~nm}$ ). 


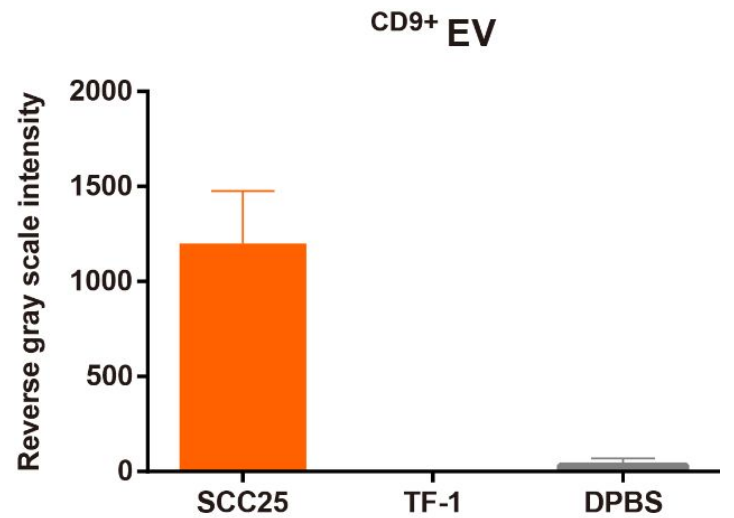

Figure S4. Validation of detection specificity with TF-1 cells. TF-1 cells were cultured for $48 \mathrm{~h}$ in RPMI medium with $10 \%$ exosome depleted FBS and $2 \mathrm{ng} / \mathrm{ml}$ recombinant human GM-CSF before collecting culture medium. The CD9 capture antibody $(2 \mu \mathrm{L}, 100 \mu \mathrm{g} / \mathrm{mL})$ were immobilized on a poly-L-lysine coated glass slide. Afterward, TF-1 cells culture medium and SCC25 cells culture medium, which was used as a positive control, were added and incubated for 4 hours at $25^{\circ} \mathrm{C}$ to finish detection (DPBS as a negative control). 

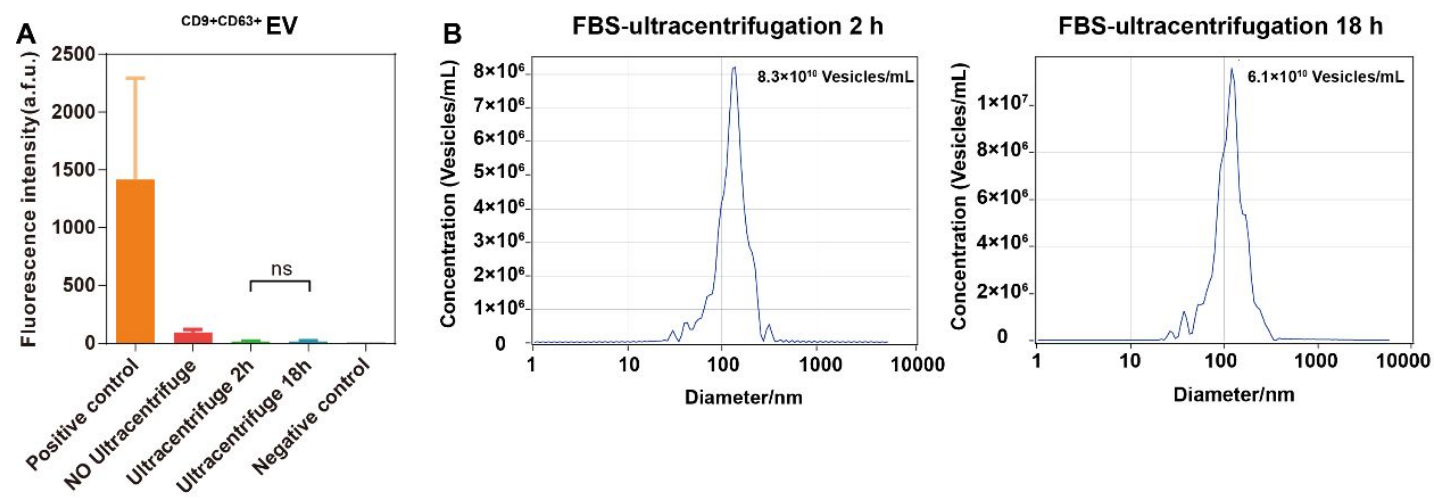

Figure S5. A comparison of exosome-depleted FBS at different ultracentrifuge times. (A) Compared with the raw FBS, the number of ${ }^{\mathrm{CD}}{ }^{+}{ }^{+} \mathrm{CD} 63+\mathrm{EVs}$ was significantly reduced after ultracentrifugation for 2 hours. However, there is no significant difference between the FBS ultracentrifuged for 2 hours and 18 hours. SCC25 cell culture medium was used as a positive control and DPBS was served as a negative control. SCC25 cells were cultured in MEM (Hyclone, America) complete medium containing $10 \%(\mathrm{v} / \mathrm{v})$ fetal bovine serum (PAN, Germany), which was ultracentrifuged for $2 \mathrm{~h}$ at $100,000 \times \mathrm{g}$ (Type $90 \mathrm{Ti}$ rotor, Beckman Coulter) at $4{ }^{\circ} \mathrm{C}$ to deplete exosomes, $100 \mathrm{U} / \mathrm{mL}$ of penicillin G sodium and 100 $\mathrm{U} / \mathrm{mL}$ of streptomycin (Hyclone, America) and 1\% (v/v) MEM Non-Essential Amino Acid (Life Technologies, America). SCC25 cells were cultured $48 \mathrm{~h}$ in a complete medium. Then, the culture medium was centrifuged for 15 min at $500 \times \mathrm{g}$ and $25 \mathrm{~min}$ at $2500 \times \mathrm{g}$ to deplete cells and cell debris, which would be used as a positive control. (B) NTA evaluation of the FBS ultracentrifuged for $2 \mathrm{~h}$ and $18 \mathrm{~h}$ revealed that particle concentration of them are $8.3^{*} 10^{10}$ vesicles $/ \mathrm{mL}, 6.1 * 10^{10}$ vesicles $/ \mathrm{mL}$, respectively. 

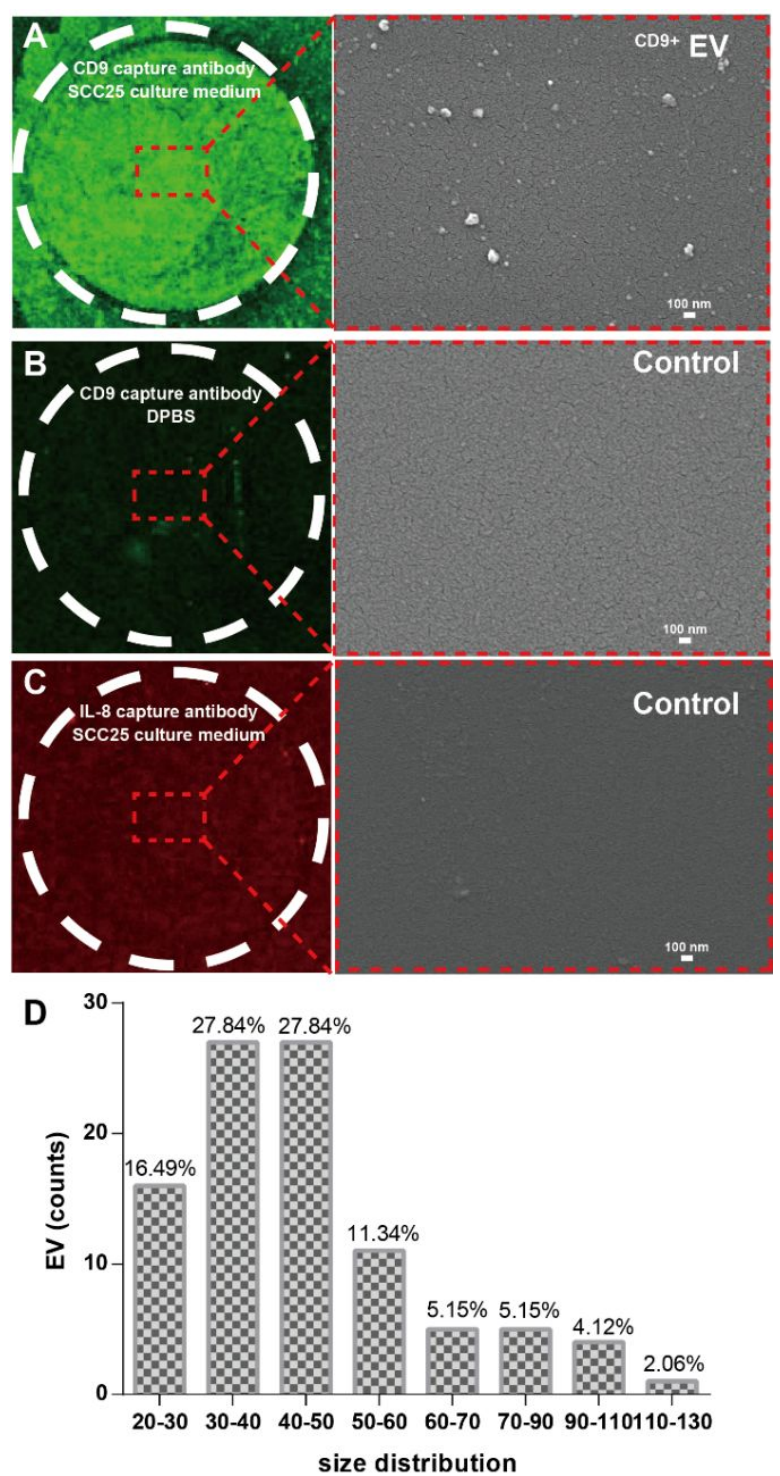

Figure S6. SEM characterization of antibody captured EVs (fluorescence detection region). (A) SEM image confirmed EVs were captured successfully on the antibody-coated spot (scale bar is $100 \mathrm{~nm}$ ). The CD9 capture antibody $(2 \mu \mathrm{L}, 100$ $\mu \mathrm{g} / \mathrm{mL}$ ) was immobilized on a poly-L-lysine coated glass slide forming a circular region. SCC25 culture medium containing EVs was added and incubated overnight. After blocking and washing, the captured EVs were fixed with $2.5 \%$ glutaraldehyde in PBS for $2 \mathrm{~h}$ and rinsed for $3 \times 10$ minutes with DPBS. The captured EVs were dehydrated in a concentration gradient of ethanol $(30 \%, 50 \%, 70 \%, 95 \%$, and $100 \%)$ for $2 \times 10 \mathrm{~min}$ at each step. After drying, the glass slide was coated with $2 \mathrm{~nm}$ platinum. The captured EVs were then imaged with JMS-7800F SEM at $3 \mathrm{kV}$. In the rectangular area of $3500 \mathrm{~nm} * 2500 \mathrm{~nm}$, a total of $96^{\mathrm{CD} 9+} \mathrm{EVs}$ were captured. Therefore, the capture capacity of the glass slide is 11 vesicles $/ \mu \mathrm{m}^{2}$, setting the upper limit of detectable density. As a positive control, another parallel experiment was carried out simultaneously. After blocking and washing, the glass slide was incubated with CD9-PE to form a fluorescence region as a positive control. The fluorescence signal intensity was read by GenePix 4300A and analyzed by GenePix Pro 7. (B) The CD9 capture antibody was immobilized on a poly-L-lysine coated glass slide and DPBS as a 
control sample were added and incubated. The following steps were the same as (A). (C) SEM image confirmed EVs and cell debris were not captured by IL-8 capture antibody. (D)The size and distribution of EVs captured on the spot.

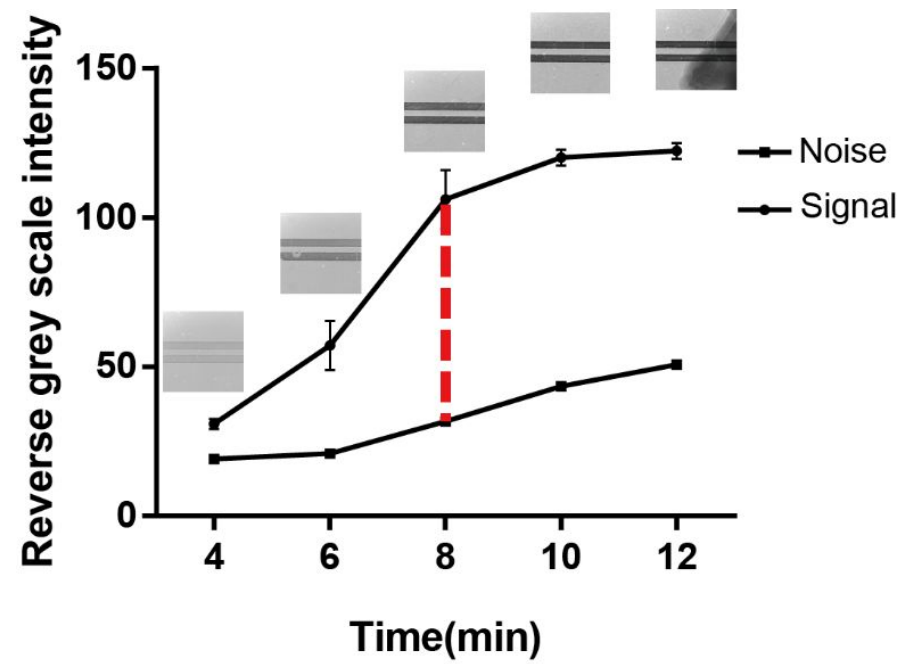

Figure S7. The time-course monitoring of silver staining showed that the largest $\mathrm{S} / \mathrm{N}$ was obtained after 8 min of silver enhancement. 


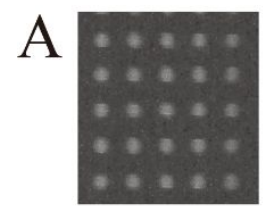

$60 \mu \mathrm{m}$

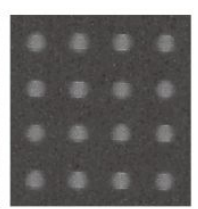

$80 \mu \mathrm{m}$

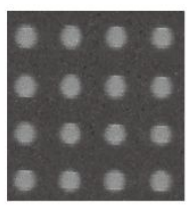

$100 \mu \mathrm{m}$

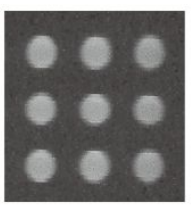

$150 \mu \mathrm{m}$

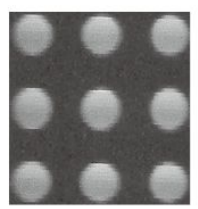

$200 \mu \mathrm{m}$

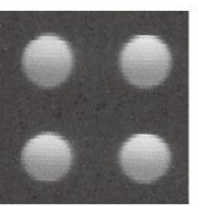

$250 \mu \mathrm{m}$

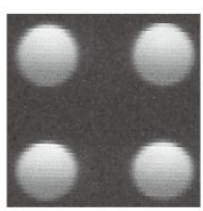

$300 \mu \mathrm{m}$
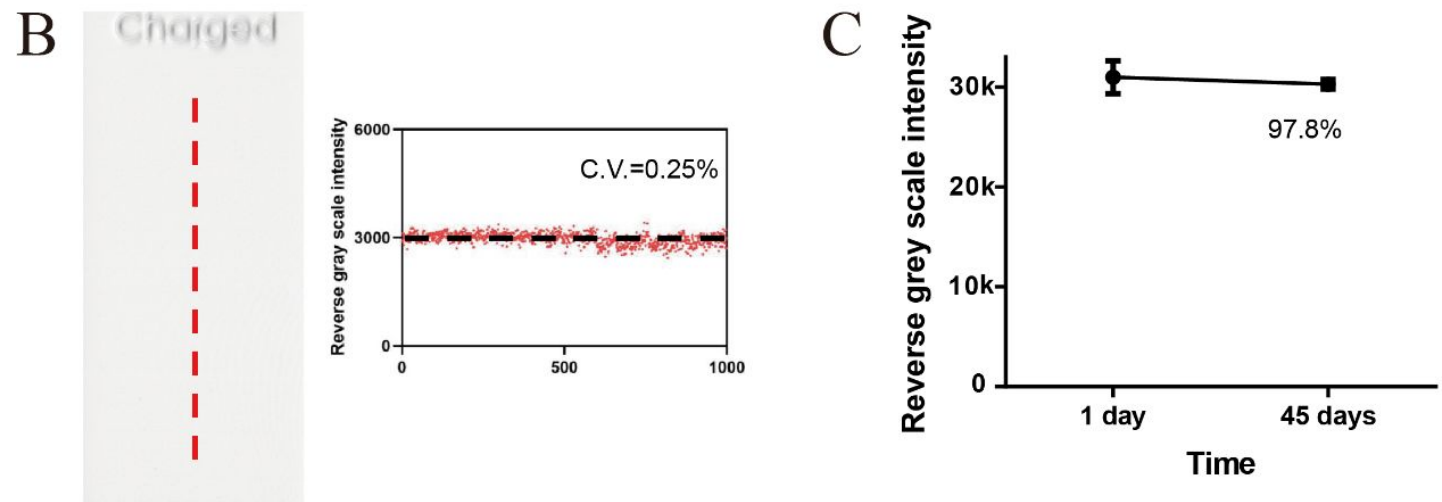

Figure S8. Characterization of desktop scanner for detection. (A) Scanning PDMS microwells with different diameters $(60,80,100,150,200,250,300 \mu \mathrm{m})$ to determine the smallest diameter of microwell to be used for detection with the scanner. (B) Scanning the bare glass slide reveals that the variance in the background light of the scanner is $0.25 \%$. (C) Signal stability characterization revealed that the silver staining signal was still $97.8 \%$ of the initial inverse grayscale intensity after 45 days of storage under normal conditions (temperature, pressure). The experimental procedures were as follows: The IL-8 capture antibodies were immobilized on a poly-L-lysine coated glass slide by incubating at $4{ }^{\circ} \mathrm{C}$ in a wet box overnight. After blocking and washing, $10 \mathrm{ng} / \mathrm{mL}$ IL-8 recombinant protein was added and incubated for $4 \mathrm{~h}$ at $25^{\circ} \mathrm{C}$. After that, the glass slide was incubated with IL- 8 biotinylated detection antibody for $1 \mathrm{~h}$, streptavidin labeled gold nanoparticles for $30 \mathrm{~min}$, and the silver enhancer solution for $8 \mathrm{~min}$. After that, the glass slide was washed with $0.05 \%$ $(\mathrm{v} / \mathrm{v})$ tween 20 in DI water to stop the reaction. The glass slide was spun dry in a slide centrifuge and imaged with an Epson V19 desktop scanner. After 45 days' storage, the glass slide was imaged again, which revealed that the inverse grayscale intensity of the silver staining signal was $96 \%$ of the initial intensity. 

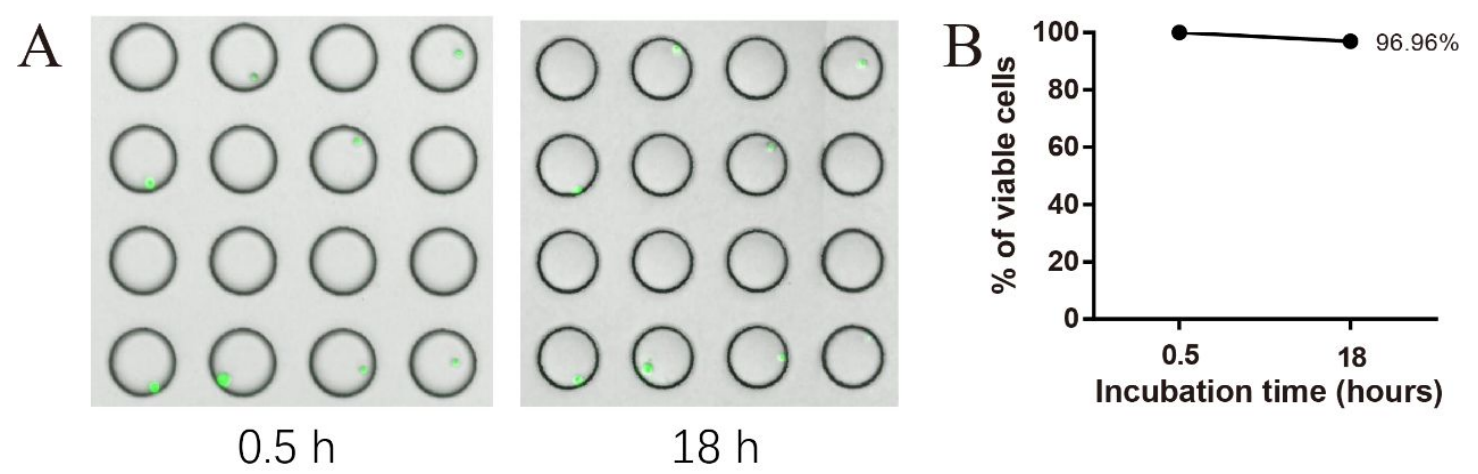

Figure S9. Cell viability characterization. The SCC25 cells stained with cell tracker dye were pipetted onto a hydrophilic PDMS microchip and incubated for $18 \mathrm{~h}$. (A) Image of the same cells at $0.5 \mathrm{~h}$ and $18 \mathrm{~h}$ after incubation. (B) $\sim 97 \%$ of trapped cells were still alive after $18 \mathrm{hrs}$ ' incubation. 


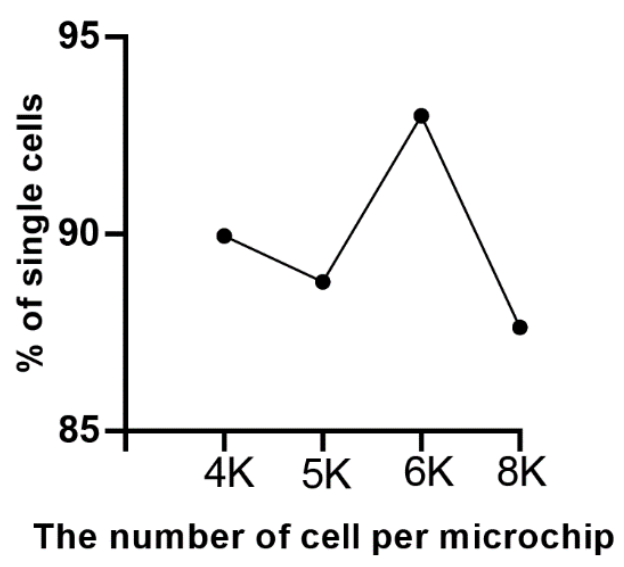

Figure S10. Cell density optimization. We pipetted the different numbers of cells $(4,000 ; 5,000 ; 6000 ; 8,000$ cells $)$ stained with Calcein-AM onto a hydrophilic treated PDMS microchip, respectively. The glass slide and PDMS microchip would be tightly clamped, and single cells were trapped inside microwells. Subsequently, the microchip was imaged with the Nikon Eclipse TiE microscope to record the number of cells captured in each microwell. We calculated the percentage of microwells with single cells related to the microwells with cells on each chip. Moreover, we found that the pipetting of 6000 cells could result in the most significant percentage of single cells. 


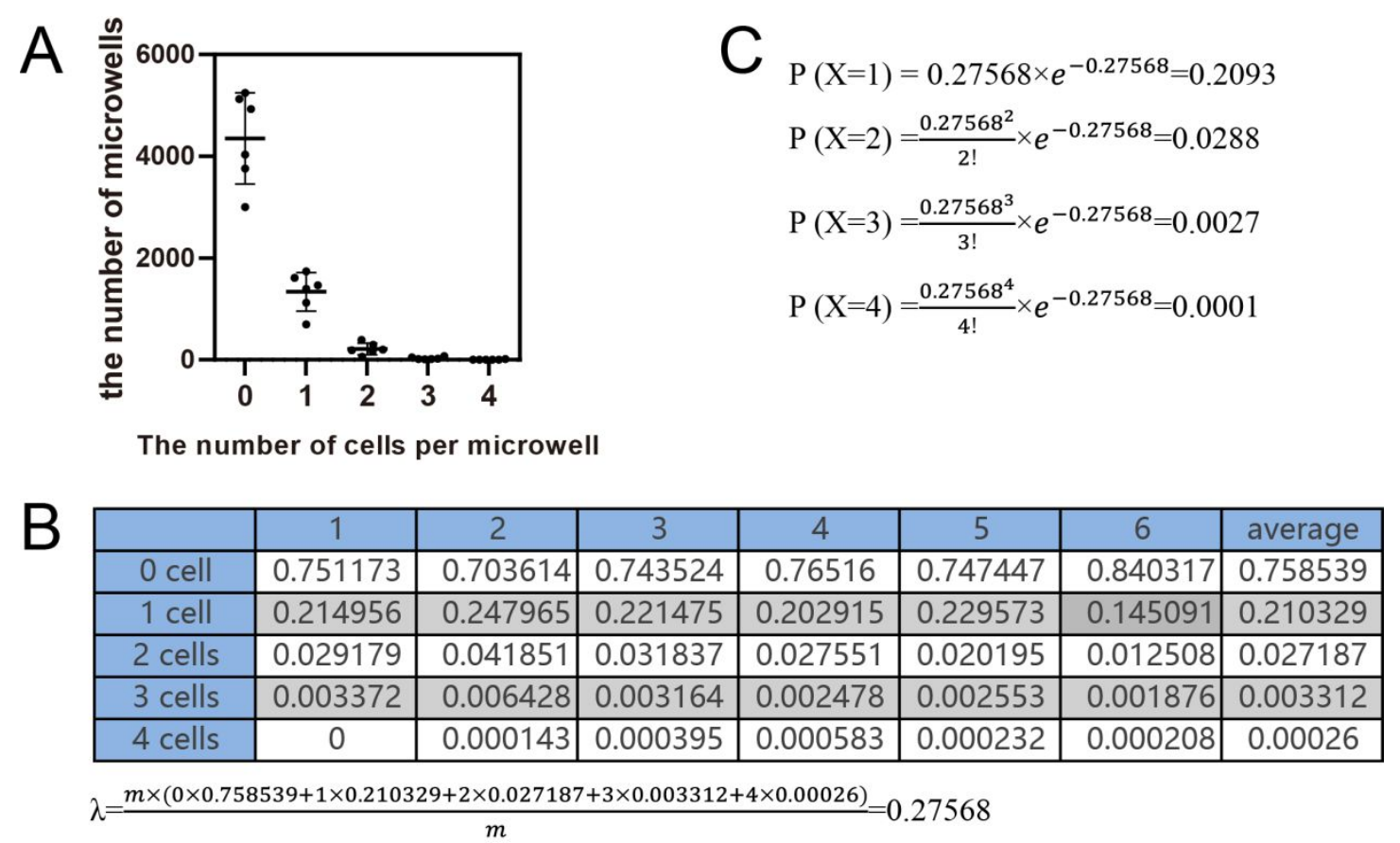

Figure S11. Calculation of the theoretical value of the number of captured cells. (A) The distribution of the number of cells captured on a microchip. (B) We calculated $\lambda=0.27568$ based on the experimental distribution probability of 0,1 , 2,3 , or 4 cells in each microwell. " $\mathrm{m}$ " is defined as the number of microwells analyzed. (C) According to the Equation of Poisson distribution, we can calculate the theoretical value of the probability of capturing 1, 2, 3, or 4 cells in microwells. 

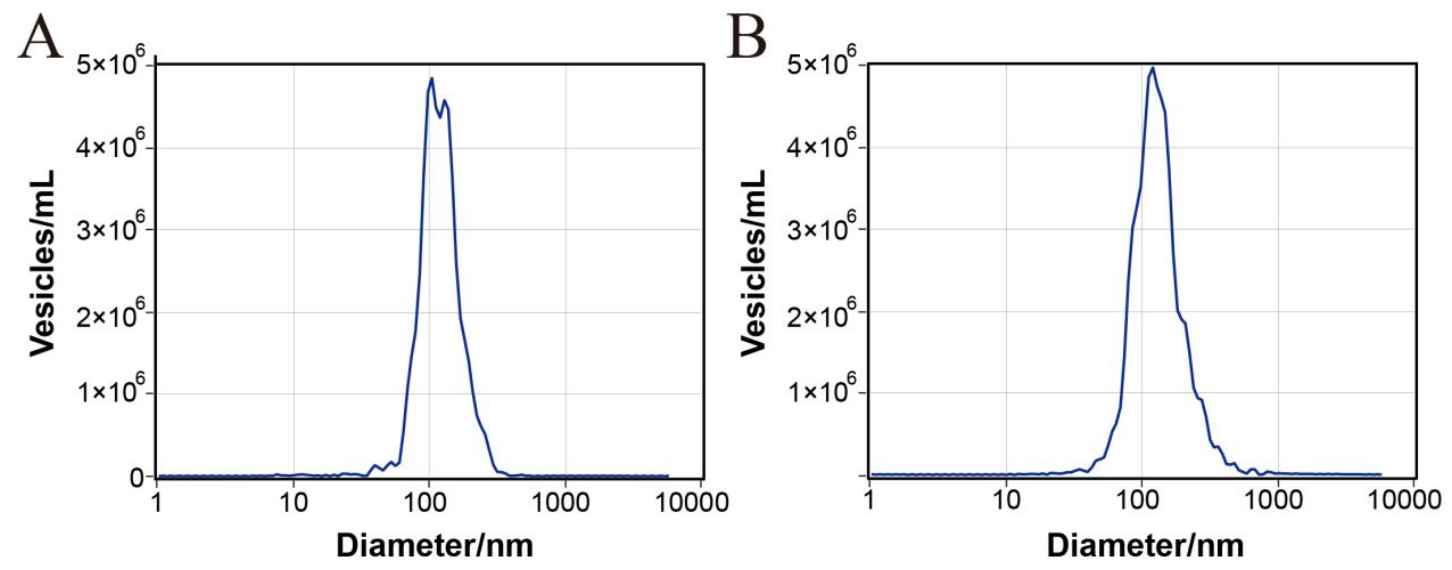

Figure S12. Characterization of EV concentration in culture medium with NTA (Particle Metrix ZetaView ${ }^{\circledR}$, Germany. Measurement Mode: Size Distribution 3 Cycles 11 Positions). (A) NTA measurement of cell medium from SCC25 cells. (B) NTA curve of concentrated culture medium. 

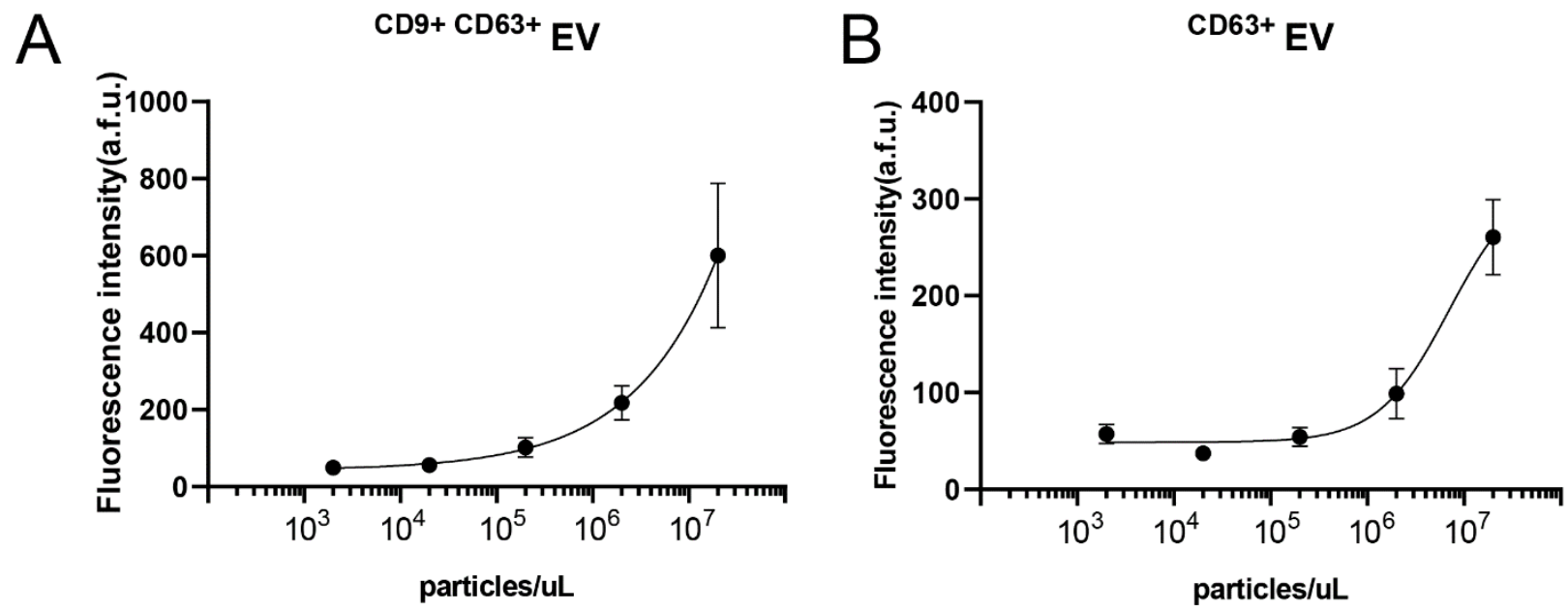

Figure S13. The calibration curves for ${ }^{\mathrm{CD} 9+\mathrm{CD} 63+} \mathrm{EVs}(\mathrm{A})$ and ${ }^{\mathrm{CD} 63+} \mathrm{EVs}$ (B) using EV standard (Biovision, US) by immunofluorescence. The CD9 and CD63 capture antibodies were immobilized on a poly-L-lysine coated glass slide to form an antibody stripe. EV standards with different concentrations were added and incubated overnight. After blocking and washing, the glass slide was incubated with biotin-CD63 for $1 \mathrm{~h}$ and stained with streptavidin-APC for $0.5 \mathrm{~h}$. The glass slide was washed with DPBS, DPBS/DI water(v/v=1/1), and DI water sequentially. Then it was spun dry in a slide centrifuge. The fluorescence results were read by GenePix 4300A and analyzed with GenePix Pro7. And the fluorescence intensity averaged from 6 spots. 


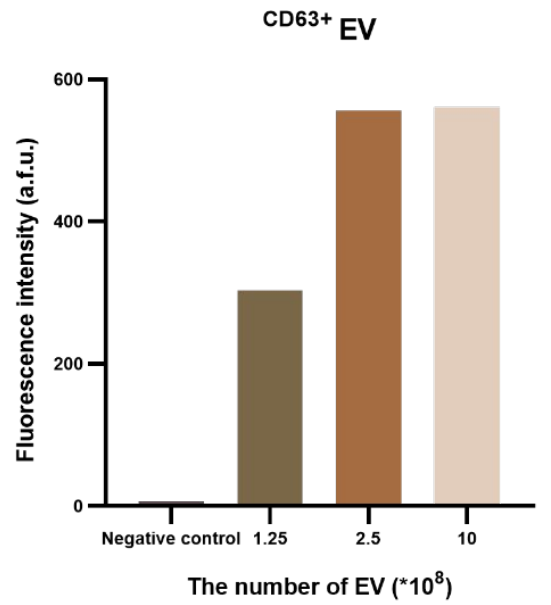

Figure S14: Characterization of the maximum EV capturing capacity of the CD63 antibody glass slide. The solution containing varying amounts of EVs $\left(1.25^{*} 10^{8} \sim 1 * 10^{9}\right)$ was pipetted onto the CD63 antibody glass slide, the biotinylated anti-CD63 antibody, and streptavidin-APC for immunofluorescence detection. DPBS was served as a negative control. 
Table S1. Summary of reagents used.

\begin{tabular}{lll}
\hline Capture antibody & Company & Catalog number \\
\hline Anti-Hu CD9 & Millipore & CBL162 \\
Anti-Hu CD63 & Millipore & CBL553 \\
Anti-Hu CD81 & Novus & NB100-65805 \\
Biotin-Anti-Hu CD63 & Biolegend & $353017-50$ \\
Streptavidin-gold nanoparticles & eBioscience & S9059 \\
Silver Enhance Kit & Sigma-Aldrich & SE-100 \\
Streptavidin-APC & Thermo & $17-4317-82$ \\
\hline
\end{tabular}

Table S2. The patients' medical records.

\begin{tabular}{|c|c|c|c|c|c|c|}
\hline Patient & Gender & Age & $\begin{array}{l}\text { Tumor } \\
\text { type }\end{array}$ & Location & Differentiation & Metastasis \\
\hline 1 & Male & 62 & OSCC & Gingiva & High & No \\
\hline 2 & Male & 55 & OSCC & Mouth floor & High & Yes (cervical lymph node) \\
\hline
\end{tabular}

